\title{
Simple evaluation method of adhesive failure criterion in multiaxial stress states by uniaxial tensile tests
}

\author{
Masato IIMORI*, Hiro TANAKA*, Mayuko KIMURA*, Yoji SHIBUTANI*,** and Yang LIU*** \\ *Department of Mechanical Engineering, Osaka University \\ 2-1 Yamadaoka, Suita, Osaka 565-0871, Japan \\ E-mail: sibutani@mech.eng.osaka-u.ac.jp \\ ${ }^{* *}$ Nanotechnology Program, Vietnam Japan University \\ My Dinh Campus, Luu Huu Phuoc Road, My Dinh 1 Ward, Nam Tu Liem District, Ha Noi, Vietnam \\ ***Department of Mechanical Engineering, Sojo University \\ 4-22-1 Ikeda, Nishi-ku, Kumamoto 860-0082, Japan
}

Received: 2 November 2017; Revised: 25 December 2017; Accepted: 15 January 2018

\begin{abstract}
We propose a simple tensile test with which to identify the parameters that constitute the failure criterion of an adhesive in a multiaxial stress state. In this paper, we define the failure criterion as the proportional limit of load-displacement curves obtained from experiments. We first introduced the failure criterion based on the first invariant of the stress tensor and the second invariant of the deviatoric stress tensor. To determine the unknown parameters of the failure criterion, two experiments were performed. The first was a tensile shear test for bonded plate structures to obtain the strength of the adhesive material in simple shear deformation and assess how the strength depends on the strain rate and adhesion layer thickness. We subsequently performed a uniaxial tensile test for circular pipe specimens bonded by the same adhesive. The pipe specimens had inclined cutting surfaces for the purpose of measuring adhesive failure points in a multiaxial stress state. Using the failure criterion with the developed tensile test, we next evaluated the adhesive strength of the pipe specimens with reference thickness of $h^{*} \sim 0.4 \mathrm{~mm}$. Formulating a scale function $\delta$ as a reference of $h^{*}$, we calculated the failure magnitudes of pipe specimens with different thicknesses $h$. As a result, we obtained the power law of $\delta$ $\propto h^{-0.79}$, which allows us to extend the failure criterion to different thicknesses of the adhesive layer.
\end{abstract}

Keywords : Adhesive, Failure criterion, Multiaxial stress state, Thickness dependence, Strain rate dependence

\section{Introduction}

There has been a great effort in recent years to produce lightweight cars and thus reduce energy consumption and $\mathrm{CO}_{2}$ emissions. Multi-material design, where components are made from materials of appropriate stiffness and strength, is one strategy for lightening cars. To this end, several methods of joining dissimilar materials, such as laser welding (Katayama and Kawahito, 2008), friction stir spot welding (Liyanage et al., 2009), mechanical fastening with bolts and adhesion (Kawasaki et al., 2016), have been developed. In particular, adhesive bonding is applicable to joints between various combinations of dissimilar materials, unlike the case for welding, and most adhesive materials are lighter than mechanical fastenings.

Adhesive failure assessment based on a multiaxial stress state is valid for lightweight constructions with multiple materials because a multiaxial stress state is expected to exist at the interface in practical design. At present, there are a quadratic stress criterion (Brewer and Lagace, 1988) and other failure criteria based on the first invariant of the stress tensor and the second invariant of the deviatoric stress tensor (Dolev and Ishai, 1981, Adams et al., 1986, Richardson et al., 2003, Mahnken and Schlimmer, 2005). However, most of these criteria have unknown parameters that depend on the material properties of adhesives. We thus need to identify the parameters from experiments prior to employing these criteria.

There are lap joint tests that measure the strength of the adhesive layer; e.g., single-lap shear tests (Ikegami and 
Sugibayashi, 1987, Adams, 1989, Pinto et al., 2009) and peel tests (Kaelble, 1992, Thouless and Jensen, 1992). However, these measurements are not appropriate for fitting a failure criterion that regards stress as uniform in the adhesive layer because they allow a stress singularity at the free edge. A napkin-ring specimen has been proposed to avoid the stress concentration effect (Bossler et al., 1968, Liechti and Hayashi, 1989, Chai, 1993, 2004). A uniform shear stress state is generated in the adhesive layer of the ring specimen subjected to torsion. That is to say, a tensile/torque test method with a napkin-ring specimen has been developed so that the parameters of the failure criterion for multiaxial stress states can be calculated from experimental results to tune the ratio of applied tension and torque (Richardson et al., 2003, Mahnken and Schlimmer, 2005, Spaggiari, et al., 2013, Teutenberg, et al., 2013). Although this method can evaluate a failure criterion without a free-edge effect, a highly accurate biaxial testing machine, which few research institutions might possess, is required. A modified Arcan test has been also developed to investigate the strength of the bonded assembly under multiaxial stress states (Cognard et al., 2005) and this test method enables to limit the free-edge effect by modifying the geometry near the free edge (Cognard et al., 2006, Cognard, 2008).

This paper presents a failure criterion of an adhesive in multiaxial stress states and a simple evaluation method that allows different multiaxial ratios of tensile and shear stresses to be applied in the adhesive layer of the bonded pipe specimen subjected to only uniaxial tension. We carry out tensile tests on specially processed pipe specimens, which have an inclining bonded surface, to yield the tensile and shear stress state in the adhesive zone, and then determine the unknown parameters of failure criterion for the multiaxial stress states. We adopt the proportional limit as the threshold of its failure in the present research, which is much utilized from the viewpoint of an engineering design of adhesive structures.

We next introduce a scale function to assess the thickness dependence on the failure magnitude of the pipe specimens because the strength of the adhesive structure in general varies with the thickness of the adhesive layer (Kaelble, 1992, da Silva et al., 2006). Finally, we extend the failure criterion to different thickness of the adhesive layer using the power law between the scale function and adhesive thickness.

\section{Failure function of the adhesive in multiaxial stress states}

To realize multiaxial stress states by a uniaxial tensile test, we develop a tensile test method using circular pipe specimens that have inclined cutting surfaces (see Fig. 1). According to the inclination angle, this specimen allows the different ratio of tensile and shear stress to be applied in adhesive layer of the bonded pipe specimen subjected to only uniaxial tension as shown Fig. 1(b).

We established two cylindrical coordinate systems as shown in Fig. 1(a). The coordinates $\left(r^{\prime}, \theta^{\prime}, z^{\prime}\right)$ are transformed from $(r, \theta, z)$ in terms of the angle $\varphi$ between $r \theta$ and $r^{\prime} \theta^{\prime}$ planes: the second plane is parallel to the cutting surface as shown in Fig. 1(a).
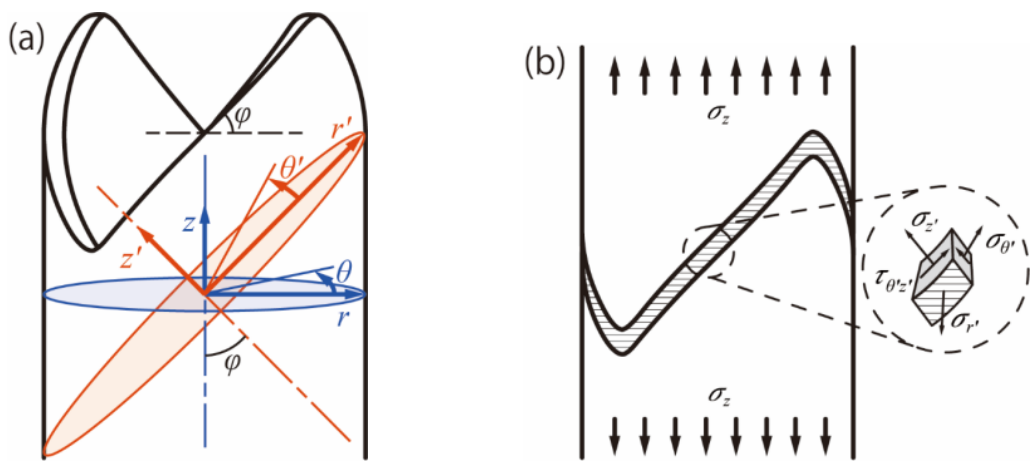

Fig. 1 (a) Coordinate transformation of the cylindrical coordinate system from $(r, \theta, z)$ to $\left(r^{\prime}, \theta^{\prime}, z^{\prime}\right)$ in terms of angle $\varphi$. (b) Stress tensor components based on the $\left(r^{\prime}, \theta^{\prime}, z^{\prime}\right)$ coordinate system in the adhesive layer.

In Fig. 1(b), the normal strain acting in radial and circumferential directions along the cutting surface in the adhesive layer is restricted by mother material surfaces because the stiffness of the adhesive is much less than that of the mother material. We thus assume that 


$$
\frac{\varepsilon_{r^{\prime}}}{\varepsilon_{z^{\prime}}} \approx 0, \frac{\varepsilon_{\theta^{\prime}}}{\varepsilon_{z^{\prime}}} \approx 0
$$

This assumption has been certified using finite element simulations for the Poisson's ratio with range of 0.2 to 0.4 (see Appendix A). Constitutive equations are derived from Eq. (1):

$$
\sigma_{r^{\prime}}=\sigma_{\theta^{\prime}}=\frac{E v}{(1+v)(1-2 v)} \varepsilon_{z^{\prime}}, \quad \sigma_{z^{\prime}}=\frac{E(1-v)}{(1+v)(1-2 v)} \varepsilon_{z^{\prime}} .
$$

According to Eq. (2), $\sigma_{r^{\prime}}$ and $\sigma_{\theta^{\prime}}$ can be expressed as a function of $\sigma_{z^{\prime}}$ :

$$
\sigma_{r^{\prime}}=\sigma_{\theta^{\prime}}=\frac{v}{1-v} \sigma_{z^{\prime}}
$$

The first invariant of stress tensor $I_{1}$ and the second invariant of deviatoric stress tensor $J_{2}$ are then calculated, respectively, as

$$
\begin{aligned}
& I_{1}=\sigma_{r^{\prime}}+\sigma_{\theta^{\prime}}+\sigma_{z^{\prime}}=\frac{1+v}{1-v} \sigma_{z^{\prime}}, \\
& J_{2}=\frac{1}{2}\left\{\left(\sigma_{r^{\prime}}^{2}+\sigma_{\theta^{\prime}}^{2}+\sigma_{z^{\prime}}^{2}+2 \tau_{r^{\prime} \theta^{\prime}}^{2}+2 \tau_{\theta^{\prime} z^{\prime}}^{2}+2 \tau_{z^{r^{\prime}}}^{2}\right)-\frac{1}{3} I_{1}^{2}\right\}=\frac{1}{3}\left(\frac{1-2 v}{1-v}\right)^{2} \sigma_{z^{\prime}}^{2}+\tau_{\theta^{\prime} z^{\prime}}^{2}
\end{aligned}
$$

In Eq. (5), we assume that $\tau_{r^{\prime} \theta^{\prime}}$ and $\tau_{z^{\prime} r^{\prime}}$ are infinitesimal compared with $\tau_{\theta^{\prime} z^{\prime}}$ and $\sigma_{z^{\prime}}$. Using an arbitrary angle $\varphi, \sigma_{z^{\prime}}$ and $\tau_{\theta^{\prime} z^{\prime}}$ as functions of $\sigma_{z}$ can be respectively described as

$$
\begin{aligned}
\sigma_{z^{\prime}} & =\frac{F \cos \varphi}{A / \cos \varphi}=\sigma_{z} \cos ^{2} \varphi, \\
\tau_{\theta^{\prime} z^{\prime}} & =\frac{F \sin \varphi}{A / \cos \varphi}=\sigma_{z} \sin \varphi \cos \varphi,
\end{aligned}
$$

where $F$ is a loading force and $A$ is the cross-sectional area of the pipe. Eliminating $\sigma_{z^{\prime}}$ and $\tau_{\theta^{\prime} z^{\prime}}$ by Equations (6) and (7), $I_{1}$ and $J_{2}$ have the following linear relation, the slope of which can be determined only by Poisson's ratio and the angle $\varphi$,

$$
\sqrt{J_{2}}=\left\{\frac{1-v}{1+v} \sqrt{\frac{1}{3}\left(\frac{1-2 v}{1-v}\right)^{2}+\tan ^{2} \varphi}\right\} I_{1} .
$$

The failure function proposed by Mahnken and Schlimmer (2005) is

$$
f=J_{2}+\frac{1}{3} c_{1} I_{1}+\frac{1}{3} c_{2} I_{1}^{2}+c_{3}
$$

In the case that $c_{1}=0$ and $c_{2} \neq 0$, the failure criterion proposed by Green (1972) is obtained, and Eqs. (4) and (5) reveal that it is equivalent to the quadratic delamination criterion proposed by Brewer and Lagace $(1988)$. If $c_{1}=c_{2}=0$, the failure function is equivalent to the von Mises failure criterion. Equations (4) and (5) clearly show that the failure function in Eq. (9) can be calculated with the three parameters $v, \varphi$ and $\sigma_{z}$. The parameters $\varphi$ and $\sigma_{z}$ are determined by the pipe specimens and by the results of tensile tests, respectively. Poisson's ratio $v$ of the adhesive is the sole material parameter, and in what follows, we calculate the failure function $f$ for $v=0.4$, which is the standard value for many polymeric adhesives. The effect of Poisson's ratio will be discussed in the last part of the discussion section. 


\section{Simple shear assessment}

\subsection{Preparation of plate specimens for simple shear assessment}

Before testing for multiaxial loading, we conducted simple shear tests of bonded plate specimens to obtain the adhesive strength in simple shear deformation. We prepared two plate specimens made of A5052 aluminium which were cut by laser processing machine (TruLaser 5030: rated output $6 \mathrm{~kW}$, Trumpf $\mathrm{GmbH}+\mathrm{Co}$. KG) and bonded with a commercial acrylic adhesive (Hardloc ${ }^{\mathrm{TM}}$ : M-600-08, Denka Co., Ltd.) in regions AA' and BB' as illustrated in Fig. 2(a). The plate thickness was $3 \mathrm{~mm}$ and we tuned the thickness of the adhesive layer, which ranged from 0.3 to $1.5 \mathrm{~mm}$. Figure 2(b) shows a magnified view of the adhesive layer with red circles indicating the measurement points of the adhesive thickness. We made measurements at a total of eight points on the two sides of a specimen and calculated the mean thickness $h$.
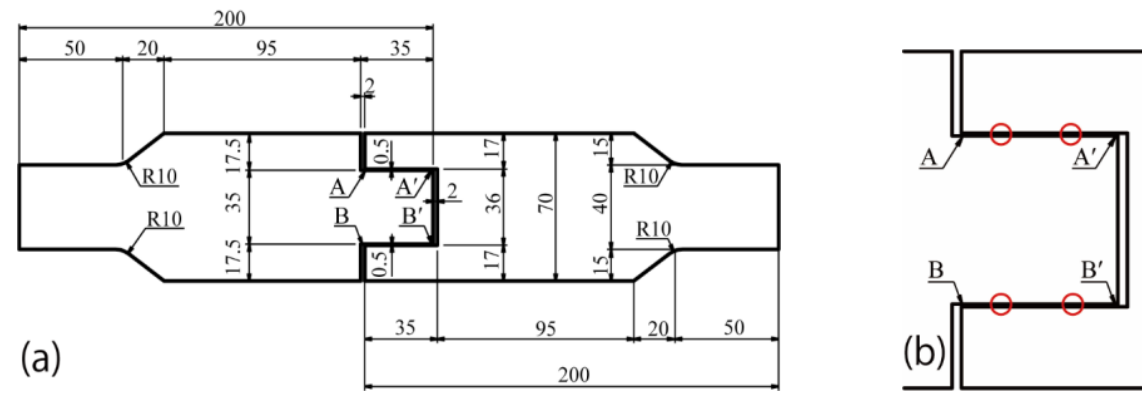

Fig. 2 Two plate specimens bonded with an adhesive in regions of AA' and BB'. (a) General view of the specimens. (b) Magnified view of the adhesive layer. Red circles indicate measurement points of the adhesive thickness.

\subsection{Tensile shear testing}

We performed a tensile shear test using a uniaxial tensile testing machine (AG-50kNX, Shimadzu Corp.) at room temperature of $293.9 \pm 0.5 \mathrm{~K}$. We selected three crosshead speeds $V_{z}(0.01,0.1$ and $1.0 \mathrm{~mm} / \mathrm{min})$ for the 13 bonded specimens with different sizes of adhesive thicknesses to evaluate strain rate dependence. In parallel with the tensile shear tests, we observed adhesive layers with a microscope (Z300PC2, Shodensha) and measured adhesive displacements in the tensile direction with image processing (see Appendix B) for getting the displacement with much higher precision than that from the crosshead of tensile machine. The flexure of the specimens was checked during the tests with strain gages that was attached to the two sides of specimens.

Figures 3(a) to (c) show the measured load-displacement curves of the specimens categorized into three groups by thicknesses of (a) $\sim 1.10 \mathrm{~mm}$, (b) $\sim 0.85 \mathrm{~mm}$ and (c) $\sim 0.60 \mathrm{~mm}$. We obtain the proportional limit $\tau$ from the load-displacement curves. From the linear parts of Fig. 3, the shear modulus of the present adhesive material is calculated and the mean value of that is $126 \mathrm{MPa}$.

\subsection{Strain rate dependence}

In this section, we aim to evaluate the strain rate dependence and thickness dependence of the strength of the bonded structure. In general, the strength of the bonded structure varies with the thickness of the adhesive layer; e.g., the strength of the single-lap shear joint decreases as the bond thickness increases. The reason considered is that the thicker adhesive layer increases stress at the interface between the adhesive and adherend (da Silva et al., 2006) and does the volume fraction of voids in the adhesive which become fracture cores. Meanwhile, the strain rate in the adhesive layer depends on the structural size of specimens when the tensile speed is fixed, which is why the stress-strain curve changes according to the viscoelastic property of the adhesive. Thus, the effective thickness dependence on their strength includes the strain rate factor in addition to the size effect of the pure bond thickness. From the results of simple shear tests, we here attempt to distinguish two factors of the adhesive shear strength, i.e., the viscoelasticity and thickness dependencies of the adhesive layer.

The strain rate $\dot{\gamma}$ is obtained by image processing with a microscope, and Figs. 4(a) and (b) show plots of log $\tau$ versus $\log \dot{\gamma}$ and $\log \tau$ versus $\log h$, respectively.

We assume that $\tau$ can be described with $\dot{\gamma}$ and $h$ :

$$
\tau=\operatorname{ch}^{\alpha} \dot{\gamma}^{\beta},
$$


where $c, \alpha$ and $\beta$ can be determined employing the least-squares method. Data fitting gives $\alpha=-0.72 \pm 0.21, \beta=0.13 \pm$ 0.04 and $c=6.31 \pm 1.69$. The fitting lines are superposed in Figs. 4(a) and (b). The comparison of $\alpha$ and $\beta$ suggests that the strain rate variation has a slight effect on the adhesive strength in the case of simple shear deformation, but it might not be dominant under the range of quasi-static loading condition which is assumed in the present research.
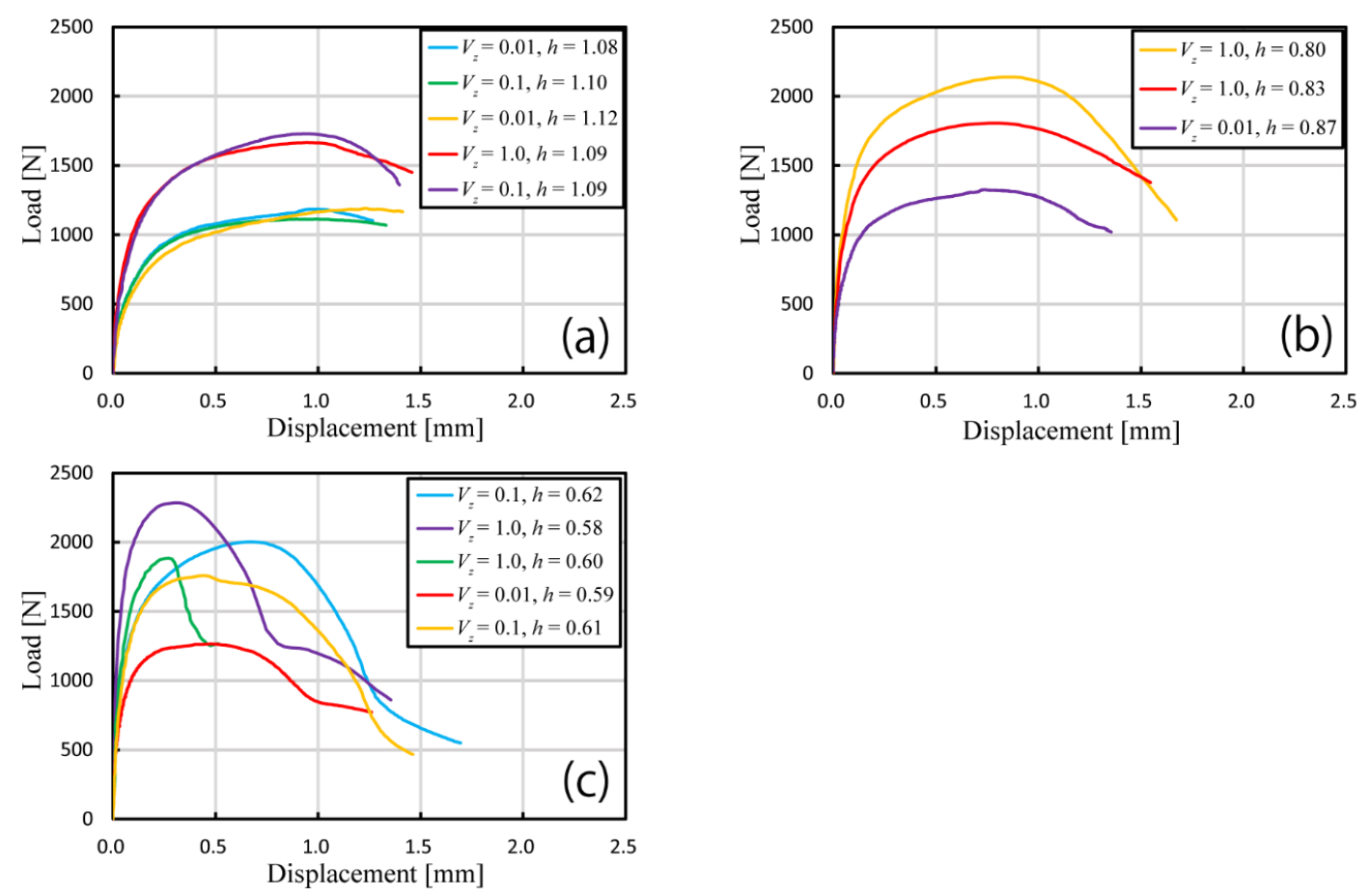

Fig. 3 Load-displacement curves of tensile shear tests, where displacements were measured with a microscope and by image processing analysis. The mean thicknesses of the adhesive layers are (a) $h \sim 1.10 \mathrm{~mm}$, (b) $h \sim$ $0.85 \mathrm{~mm}$ and (c) $h \sim 0.60 \mathrm{~mm}$.
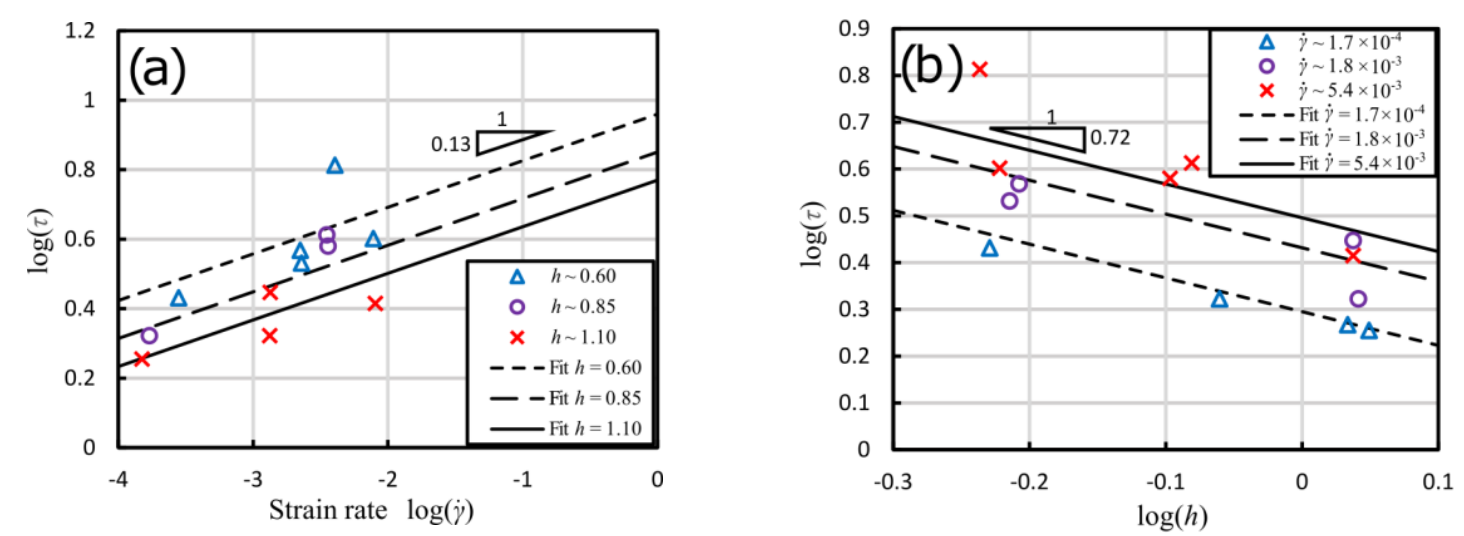

Fig. 4 Slopes of the least-squares fitting line are regarded as (a) the strain rate dependence and (b) the thickness dependence.

\section{Tensile testing for multiaxial loading}

\subsection{Preparation of pipe specimens for tensile testing}

We used circular pipe specimens that have inclined cutting surfaces to realize multiaxial stress states by a uniaxial tensile test, as shown in Fig. 5(a). The specimens made of A5052 aluminium were cut by laser processing machine (3D FABRI GEAR 220 II: rated output 4kW, Yamazaki Mazak Corp.). The selected angles between the inclining surface and horizontal plane were $0^{\circ}, 20^{\circ}, 45^{\circ}$ and $75^{\circ}$. Each pipe specimen consists of the two halves with two periodic inclination surfaces at one side; the inclination angle is denoted $\varphi$ (see Fig. 5(b)). The convex and concave portions between inclination surfaces form R2 rounds. The paired pipe halves were bonded together with the same commercial adhesive used for the tensile shear tests described in the previous section. We removed adhesive segments protruding 
from the outer surface of the pipes by polishing with sandpaper having a grit size of \#120 so that they would not affect the adhesive strength. The outer radii and thicknesses of the two pipe halves were commonly 45 and $3 \mathrm{~mm}$, respectively.

The thickness of the adhesive layer was measured with a microscope. The measurement points are indicated by eight red circles in Fig. 5(b). We calculate the mean value $h_{0}$ and standard deviation $\sigma$. In the calculations, we exclude outliers that are further than $2 \sigma$ from $h_{0}$ and recalculate the mean thickness $h$ from the remaining data in order to exclude the inaccurate thickness values measured at the remaining adhesive segments which protrude from the outer surface of the pipes.
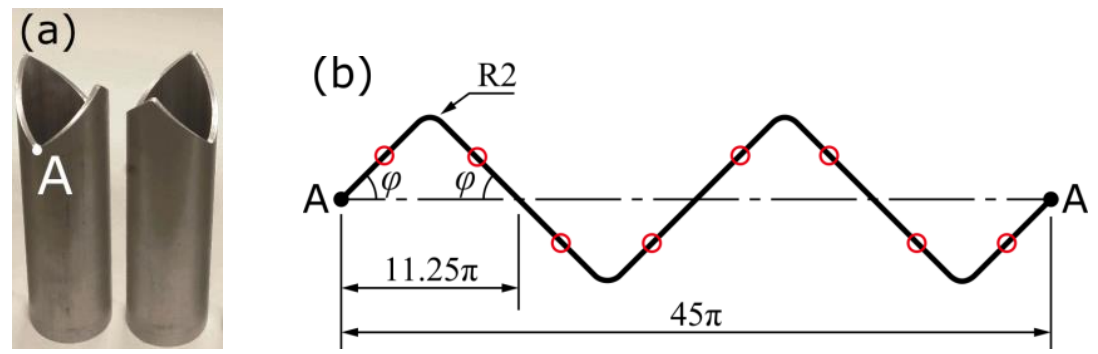

Fig. 5 (a) Example of a test specimen before adhesive bonding; the tilted cutting surface has an inclination angle $\varphi$ of $45^{\circ}$. (b) Development of the cutting surface. Red circles represent measurement points of the adhesive thickness.

\subsection{Tensile testing and results}

We carried out tensile testing using a uniaxial tensile testing machine AG-50kNX at room temperature of $296.1 \pm$ $5.7 \mathrm{~K}$. The displacement speed of the crosshead was $0.1 \mathrm{~mm} / \mathrm{min}$ throughout the tensile tests. We continued tensile testing until the tensile load dropped adequately. The flexure of the specimens was checked during the tensile tests with strain gages that was attached to the two sides of pipe specimens. The sample numbers of the four types of pipe specimens having inclination angles of $0^{\circ}, 20^{\circ}, 45^{\circ}$ and $75^{\circ}$ are $8,8,8$ and 6 , respectively.
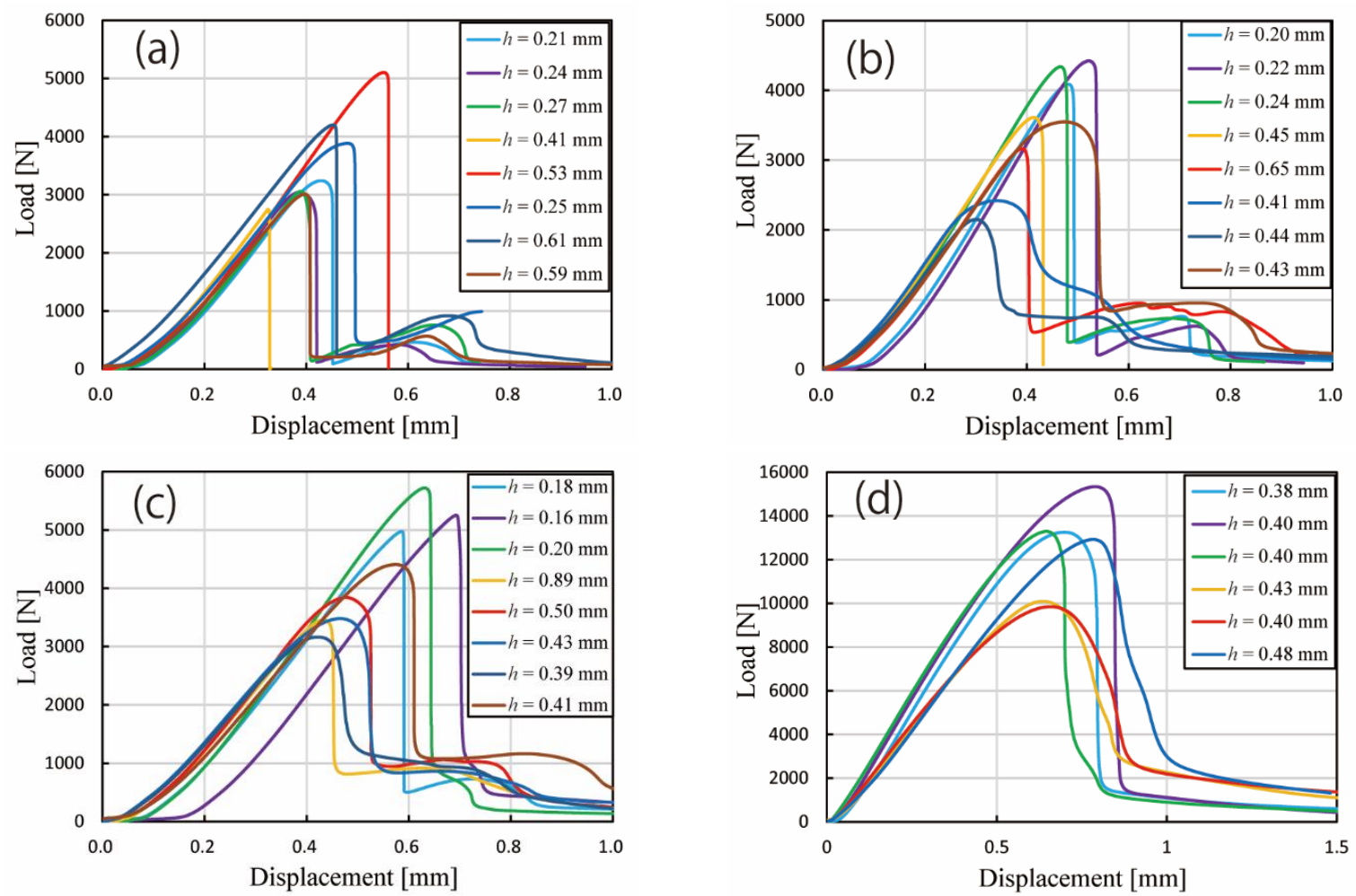

Fig. 6 Load-displacement curves obtained from tensile testing with four pipe specimens having inclination angles of (a) $0^{\circ}$, (b) $20^{\circ}$, (c) $45^{\circ}$ and (d) $75^{\circ}$. 
Load-displacement curves obtained from the tensile tests are shown in Fig. 6, where the horizontal axis indicates crosshead displacement. In the legend boxes, $h$ indicates the mean thickness of each specimen. Figure 7 shows the relationship between the mean thickness and proportional limits obtained from the load-displacement curves. In general, the fracture of adhesive layer has the viscous and plastic characterization with mean pressure dependence because the void nucleation and growth behaviors might be essential. Thus, we adopt the proportional limit as the rather clear threshold between the linear elastic and the nonlinear parts in the present research.

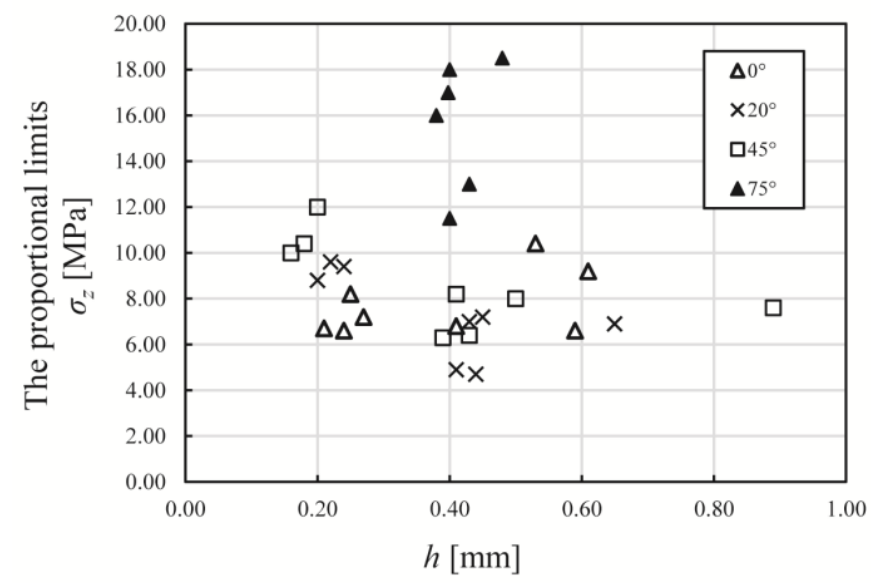

Fig. 7 Relationship between the mean thicknesses $h$ and proportional limit $\sigma_{z}$ obtained from the load-displacement curves shown in Fig. 6.

\section{Discussions on failure diagram}

The two invariant values, $I_{1}$ and $J_{2}{ }^{(1 / 2)}$, are plotted in Fig. 8, where values in the figure indicate the mean thickness $h[\mathrm{~mm}]$. Considering that the thickness of adhesive in general affects the strength of the bonded structure, we identify the failure function expressed by Eq. (9) according to the experiment results for the bonded pipe specimens with similar thicknesses of the adhesive layer $(h=0.40 \pm 0.03 \mathrm{~mm})$.

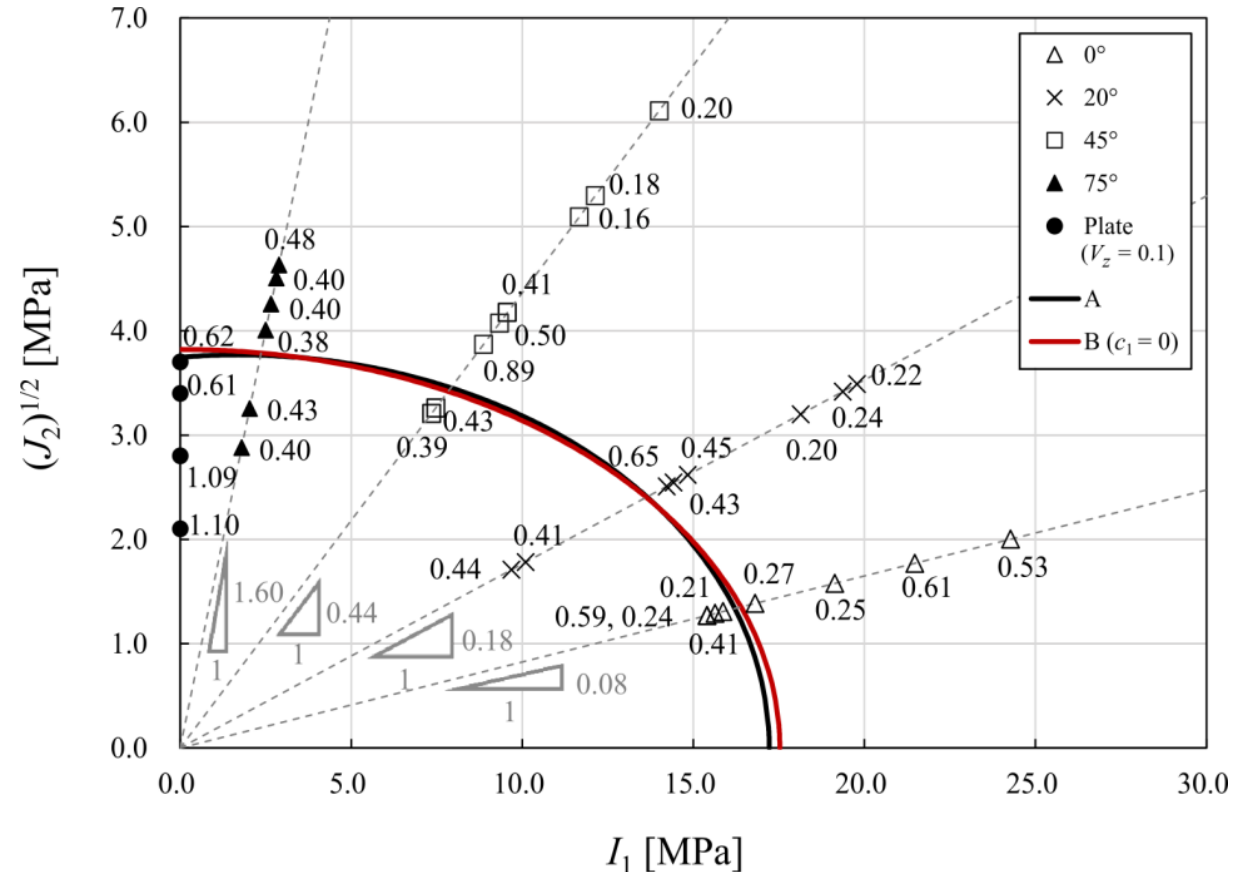

Fig. 8 Two failure criteria estimated from the experimental results of $h=0.40 \pm 0.03 \mathrm{~mm}$ and invariant values calculated from Eqs. (4) and (5). The coefficients of curve A, drawn in black, are $c_{1}=-0.60, c_{2}=0.18$ and $c_{3}=-14.04$ while those of curve $\mathrm{B}$, drawn in red, are $c_{1}=0, c_{2}=0.14$ and $c_{3}=-14.61$. 
The dashed linear lines in Fig. 8 show the relationship of Eq. (8) for the constant Poisson's ratio of 0.4 and each angle $\varphi$. Therefore, the plots with the same angle $\varphi$ are aligned on one line. Also you can find that the failure criteria should be changed according to the thickness, which will be discussed in section 5.1 .

The black curve (curve A) superposed in Fig. 8 is the fitted failure function; the determined coefficients $c_{1}, c_{2}$ and $c_{3}$ are given in Table 1. Because the coefficients have considerable error and the P-value of $c_{1}$ is much higher than a significance level of 0.05 , we recalculate the coefficients by substituting $c_{1}=0$ into Eq. (9) and employing the least-squares method; the red curve (curve B) superposed in Fig. 8 is calculated as the additional failure function and the coefficients obtained are listed in Table 2.

A comparison of curve A with curve B shows that all standard errors of parameters of curve B are less than those of curve A. This is because having $c_{1}=0$ decreases the number of fitting degrees of freedom for curve B. This implies that the number of test results with $h \sim 0.40$ is not enough to determine the three parameters using the least-squares method. If more sample data are obtained for specimens with inclination angles other than $\varphi=0^{\circ}, 20^{\circ}, 45^{\circ}$ and $75^{\circ}$, the standard error of the parameters of curve A would be smaller. Because the two curves of the sum of squared residuals are almost the same, curve B represents the experiment data as well as curve A does even though there are fewer fitting degrees of freedom for curve B than for curve A. We therefore consider that curve B is a more reliable failure criterion than curve A.

Table 1 Failure criterion of curve A (black curve) in Fig. 8. The coefficients of the failure function are determined with the experimental results of $h=0.4 \pm 0.03 \mathrm{~mm}$.

\begin{tabular}{c|c|c|c}
\hline \hline Sum of squared residuals & $c_{1}$ & $c_{2}$ & $c_{3}$ \\
\cline { 3 - 4 } 210.37 & $-0.60 \pm 4.11$ & $0.18 \pm 0.24$ & $-14.04 \pm 4.40$ \\
P-value & 0.89 & 0.49 & 0.01 \\
\hline
\end{tabular}

Table 2 Failure criterion of curve B (red curve) in Fig. 8. The coefficients of the failure function with $c_{1}=0$ are determined from the experimental results of $h=0.4 \pm 0.03 \mathrm{~mm}$.

\begin{tabular}{c|c|c|c}
\hline \hline Sum of squared residuals & $c_{1}$ & $c_{2}$ & $c_{3}$ \\
\cline { 3 - 4 } 210.94 & 0 & $0.14 \pm 0.05$ & $-14.61 \pm 1.94$ \\
P-value & - & 0.03 & $3.55 \times 10^{-5}$ \\
\hline
\end{tabular}

\subsection{Scale function from the failure criterion and thickness dependence}

While the failure function in Eq. (9) is adequate for the failure criterion of the proposed pipe specimens bonded by adhesive with the same $h$, it is not applicable to an adhesive layer with a different $h$. For example, we observe that the proportional limits corresponding to $h \sim 0.20 \mathrm{~mm}$ and $\varphi=45^{\circ}$ are away from the failure function calculated with reference to $h \sim 0.40 \mathrm{~mm}$ as shown in Fig. 8. To deal with the different thickness in a unified way, we here introduce a scale function $\delta$ (Tanaka et al., 2017):

$$
\delta=\frac{\left(I_{1}-x_{0}\right)^{2}}{a^{2}}+\frac{{\sqrt{J_{2}}}^{2}}{b^{2}}, \text { where }|a|=\sqrt{\frac{c_{1}^{2}}{4 c_{2}^{2}}-\frac{3 c_{3}}{c_{2}}},|b|=\sqrt{\frac{c_{1}^{2}}{12 c_{2}}-c_{3}} \text { and } x_{0}=-\frac{c_{1}}{2 c_{2}} .
$$

Equation (11) indicates the separation from the failure criterion curve drawn with Eq. (9) for $c_{1}=0$. We determine invariables $a=17.53, b=3.82$ and $x_{0}=0$ so that $\delta=1$ when a point $\left(I_{1}, J_{2}{ }^{(1 / 2)}\right)$ is located on curve $\mathrm{B}$ with the parameters given in Table 2. Figure 9 is a plot of $\log \delta$ versus $\log h$. The bold line is determined to pass through a point $(\log h, \log \delta)=(\log 0.4,0)$ and the slope of this line is calculated by least-squares fitting. The gradient of the line is -0.60 and $\delta$ is thus proportional to $h^{-0.60}$.

Once the thickness dependence of $\delta$ has been determined, the coefficient $c_{3}$ of the failure criteria dependent to the thickness $h$ can be calculated as

$$
c_{3}=b^{2}\left\{\frac{x_{0}^{2}}{a^{2}}-\left(\frac{h}{h^{*}}\right)^{p}\right\}
$$

where $h^{*}$ is the reference thickness of $0.4 \mathrm{~mm}$ and $p$ is the power exponent of -0.60 . From Eq. (12), the failure criteria 
for $h=0.2,0.6$ and 1.1 are obtained as Fig. 10. Figure 10 shows that the experimental results for $\varphi=0^{\circ}$ are especially far away from the proposed failure criteria.

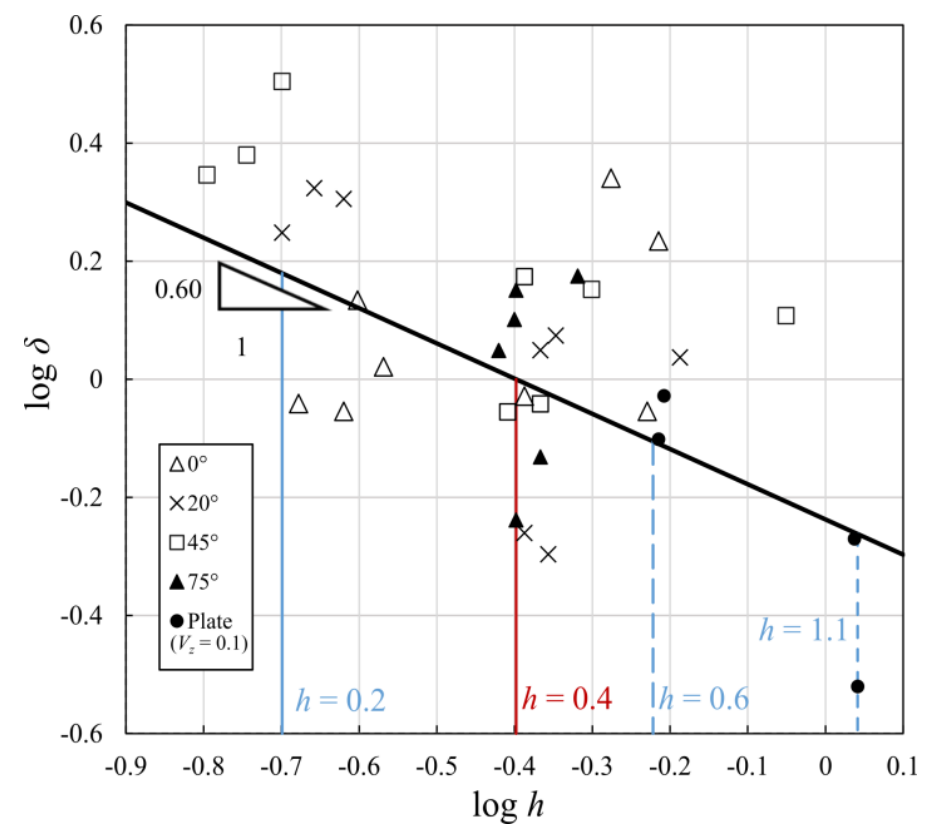

Fig. 9 Power law of the relation between the scale function $\delta$ and thickness $h$. The gradient of the bold line calculated with least-squares fitting is -0.60 and $\delta$ is thus proportional to $h^{-0.60}$.

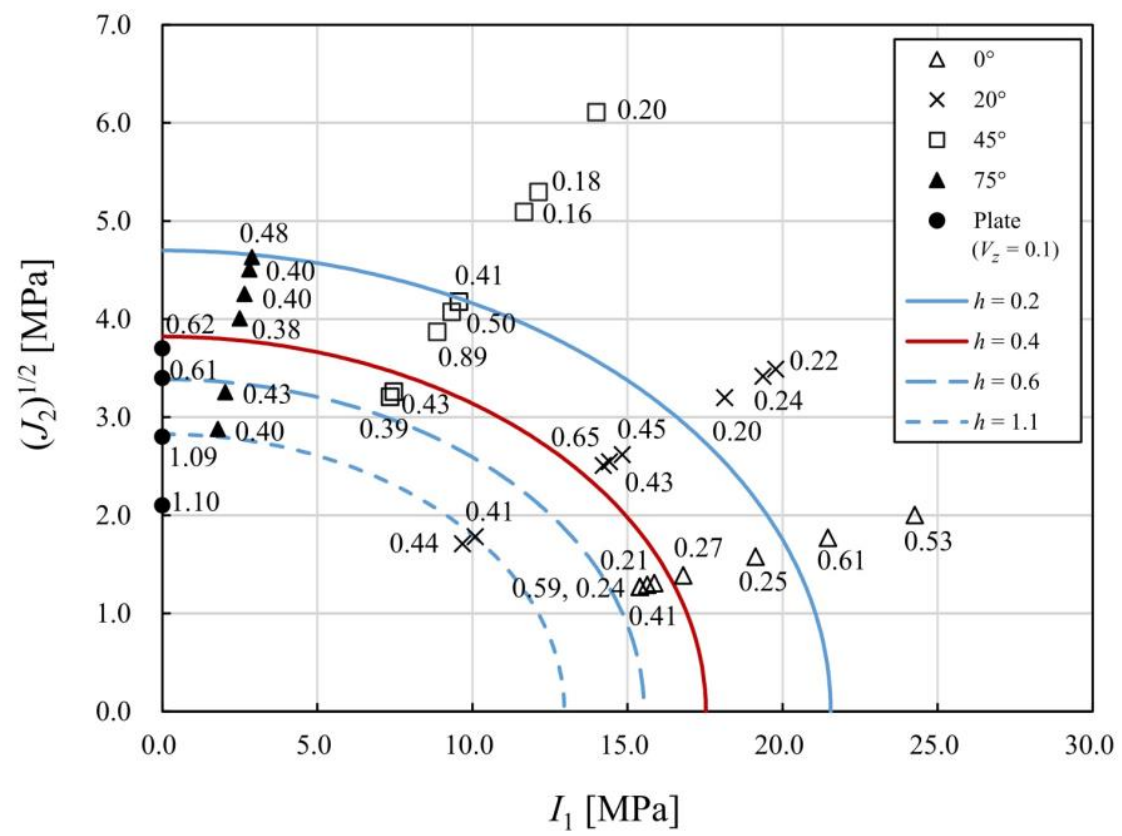

Fig. 10 The failure criteria dependent to the thickness $h$. The red curve is curve B in Fig. 8 and the blue ones are calculated from Eq. (12).

\subsection{Comparison between the failure criterion and test results}

To clarify one of the factors of the discrepancy between experimental results and the failure criterion, we calculate the fitting lines of the $\log \delta$ versus $\log h$ corresponding to each inclination angle $\varphi=0^{\circ}, 20^{\circ}, 45^{\circ}$ and the tensile shear plate as shown in Fig. 11. Each of the colored bold lines is determined to pass through a point $(\log h, \log \delta)=(\log 0.4$, $0)$ and the slopes of these lines are calculated by least-squares fitting. Note that the approximate line of $\varphi=75^{\circ}$ are not drawn in Fig. 11 because the thickness range of the plots of $\varphi=75^{\circ}$ is narrow. Figure 11 shows that the slopes of the approximate lines of $\varphi=20^{\circ}$ and $45^{\circ}$ and of the plate are in good agreement while the slope of $\varphi=0^{\circ}$ is clearly different from the other, as one of the reasons of this, we are considering that the failure of $\varphi=0^{\circ}$ is more influenced by 
the mean pressure dependence of adhesive material than that of $\varphi \neq 0^{\circ}$.

Thus, we calculate the fitting line from all data except for the case of $\varphi=0^{\circ}$ as shown in Fig. 12(a). The gradient of the fitting line is -0.79 . Substituting $p=-0.79$ into Eq. (12), the failure criteria for $h=0.2,0.6$ and 1.1 are calculated. The blue curves in Fig. 12(b) are the calculated failure criteria. To compare between the experimental results and obtained failure criteria, we identify the coefficient $c_{3}$ from the test results of $h=0.20 \pm 0.03,0.60 \pm 0.03$ and $1.10 \pm$ $0.03 \mathrm{~mm}$ by substituting $c_{1}=0$ and $c_{2}=0.14$ into Eq. (9). The average values of $c_{3}$ are listed in Table 3 . The experimental fitting curves estimated from the average values of $c_{3}$ are drawn in orange in Fig. 12(b).

Figure 12(b) and Table 3 show that the errors of $c_{3}$ between the average values of test results and Eq. (12) for $h=$ $0.60,1.10 \mathrm{~mm}$ are sufficiently small while the error for $h=0.20 \mathrm{~mm}$ cannot be ignored. One of the reasons of this large error is that the test result corresponding to $\varphi=45^{\circ}$ and $h=0.20 \mathrm{~mm}$ causes the decrease in the average value of $c_{3}$ for $h=0.20 \pm 0.03 \mathrm{~mm}$. We believe that the plot corresponding to $\varphi=45^{\circ}$ and $h=0.20 \mathrm{~mm}$ is a rare case because this plot is further away from the origin than the other plots corresponding to $h=0.16$ to $0.24 \mathrm{~mm}$. The error would be smaller when more sample data are measured for pipe specimens with $h \sim 0.20 \mathrm{~mm}$.

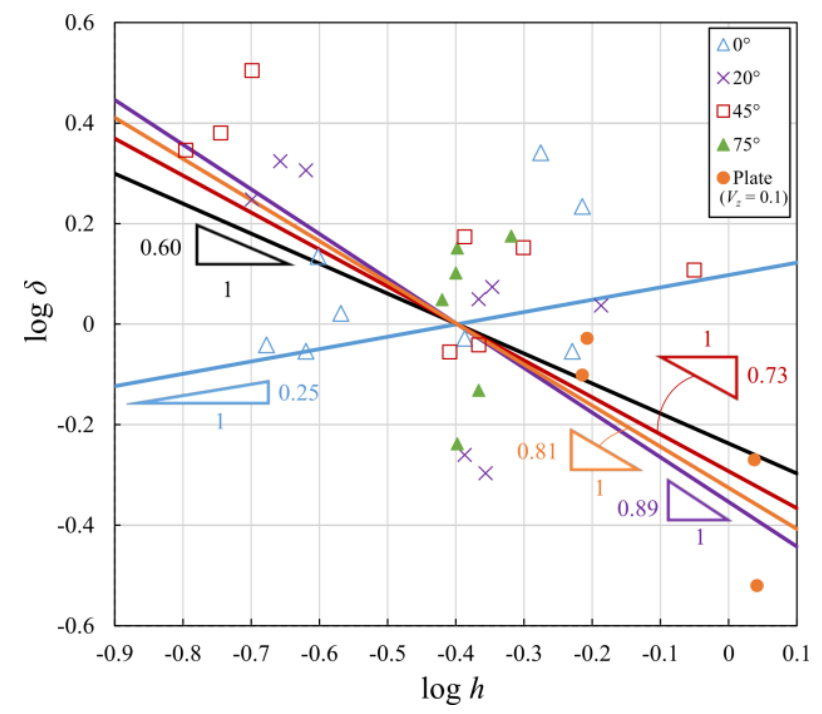

Fig. 11 The fitting lines of $\log \delta$ versus $\log h$ corresponding to the inclination angles $\left(\varphi=0^{\circ}, 20^{\circ}\right.$ and $\left.45^{\circ}\right)$ and the tensile shear plate.
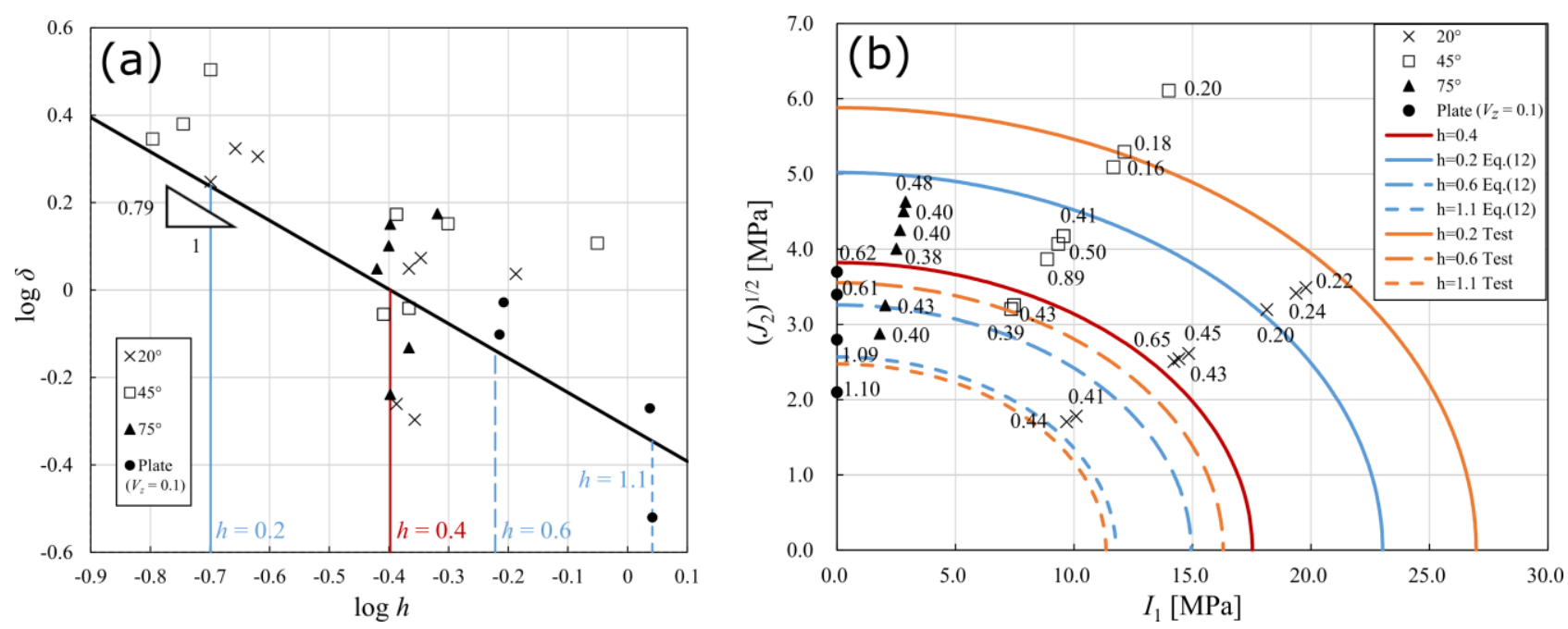

Fig. 12 (a) Power law of the relation between the scale function $\delta$ and thickness $h$ for $\varphi=20^{\circ}, 45^{\circ}, 75^{\circ}$ and the plate. (b) The failure criteria dependent to the thickness $h$ for $\varphi=20^{\circ}, 45^{\circ}, 75^{\circ}$ and the plate. The blue curves are obtained from Eq. (12) by substituting $p=-0.79$. The orange curves are the experimental fitting curves estimated from the average values of $c_{3}$ listed in Table 3 . 
Table 3 The coefficients $c_{3}$ calculated from Eq. (12) and test results.

\begin{tabular}{c|c|c|c}
\hline \hline$h[\mathrm{~mm}]$ & 0.20 & 0.60 & 1.10 \\
\hline Average value of test results & -34.59 & -12.63 & -6.13 \\
Eq. (12) & -25.20 & -10.62 & -6.59 \\
\hline
\end{tabular}

Computing the scale function (11) as described above, we assume that Poisson's ratio of the adhesive material is 0.4. Table 4 presents the other calculations of Eq. (11) with $v=0.2$ or 0.3 , from which we make sure that the value of Poisson's ration around 0.4 makes little contribution to the scale function $\delta$ and the power exponent $p$.

Table 4 Comparison of the scale functions and the power exponents $p$ for $v=0.2,0.3$ and 0.4 .

\begin{tabular}{c|c|c|c|c}
\hline \hline$v$ & $a$ & $b$ & $x_{0}$ & $p$ in Eq. (12) \\
\hline 0.40 & 17.53 & 3.82 & 0 & $-0.79 \pm 0.16$ \\
0.30 & 16.45 & 3.82 & 0 & $-0.79 \pm 0.16$ \\
0.20 & 17.41 & 3.82 & 0 & $-0.79 \pm 0.16$ \\
\hline
\end{tabular}

\section{Conclusions}

In this study, the failure criterion for the bonded structure in multiaxial stress states was evaluated with a simple testing method which we proposed. The method was applied to circular pipe specimens that had inclined bonded surfaces to obtain the tensile and shear stress in the adhesive layer with a uniaxial tensile testing machine. The unknown parameters of the failure function with reference thickness of $h^{*}=0.40$ were identified from the results of the developed tensile test and simple shear tests.

From the simple shear tests, we found out that the shear modulus of the present adhesive material was $126 \mathrm{MPa}$ and the strain rate variation might not be dominant under the range of quasi-static loading condition which is assumed in the present research.

We next introduced a scale function $\delta$ to assess the failure criterion for different thicknesses, and obtained the power law $\delta \propto h^{-0.79}$. Moreover, the failure criteria dependent to the thickness was proposed. We conclude that the proposed failure criteria would be applied to the average behavior of the test results. The manufacturing process of adhesion seems to be strongly dependent to the process control of the workplace. After getting the measurement of the cured thickness, the failure assessment in the multi-material member with adhesion would be applied for the measured thickness.

\section{Acknowledgements}

This research was based on results obtained from a future pioneering project commissioned by the New Energy and Industrial Technology Development Organization. We thank Akoo Mechanical Engineering Co., Ltd. for preparing the samples.

\section{Appendix A: Validation of the strain assumption}

We here attempt to confirm the validity of the strain assumption that the normal strain acting in radial and circumferential directions along the cutting surface of the pipe specimens are restricted by mother material. This assumption is described by Eq. (1). To obtain the normal strain values, $\varepsilon_{r^{\prime}}, \varepsilon_{\theta^{\prime}}$ and $\varepsilon_{z^{\prime}}$ in the adhesive layer, we perform a finite element simulations for the Poisson's ratio with range of 0.2 to 0.4 with the commercial FEM software (Virtual Performance Solution 2016, ESI Group).

Figure A1 shows the quarter model of the pipe specimen with outer radius of $45 \mathrm{~mm}$ and thickness of $3.0 \mathrm{~mm}$ that has an inclination angle $\varphi$ of $45^{\circ}$. The thickness of the adhesive layer is $0.5 \mathrm{~mm}$. Material parameters of the analysis model are listed as Table A1. Using the adhesive shear modulus $G=126 \mathrm{MPa}$ obtained from the simple shear tests in section 3.2, we analyzed strain distribution in adhesive layer at a tensile speed $V_{z}$ of $0.10 \mathrm{~mm} / \mathrm{min}$ until time reached $0.01 \mathrm{~s}$. Figures A2 (a) to (c) show the distribution of the strain tensor component $\varepsilon_{r}, \varepsilon_{\theta}$ and $\varepsilon_{z}$ in the adhesive layer of the model in Fig. A1. 
From $\varphi$ and the strain tensor components $\varepsilon_{r}, \varepsilon_{\theta}, \varepsilon_{z}$ and $\varepsilon_{\theta z}$, we calculated strain tensor components based on the ( $r^{\prime}$, $\theta^{\prime}, z^{\prime}$ ) coordinate system (Fig. 1 (a)) in adhesive layer except the elements at both ends of the adhesive layer which are the convex and concave portions between inclination surfaces (Fig. 5(b)). The ratio of averaged normal strains of $\varepsilon_{r^{\prime}}$ and $\varepsilon_{\theta^{\prime}}$ to the $\varepsilon_{z^{\prime}}$ are summarized into Tables A2 to A4 which correspond to the Poisson ratios of 0.4, 0.3 and 0.2. Although the ratios for $v=0.4$ reach at less than $20 \%$, the assumption might be acceptable because these values decrease as the Poisson's ratio.

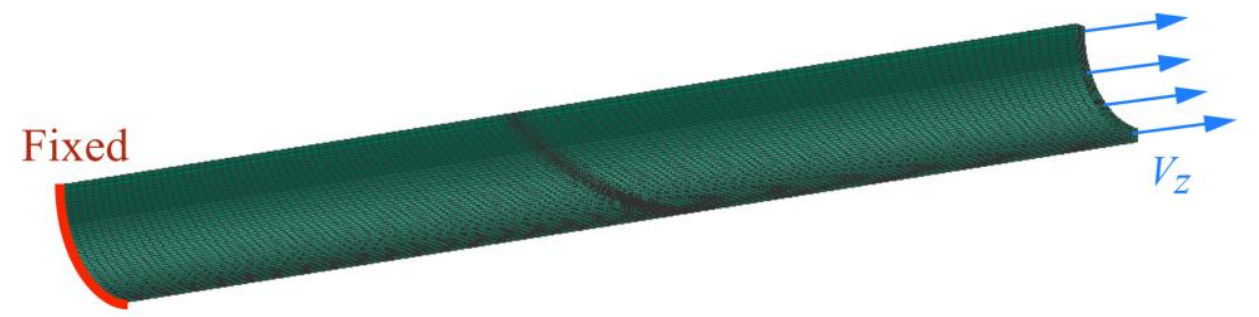

Fig. A1 The quarter analysis model of the pipe specimen with outer radius of $45 \mathrm{~mm}$ and thickness of $3.0 \mathrm{~mm}$ that has an inclination angle $\varphi=45^{\circ}$. The thickness of the adhesive $h=0.5 \mathrm{~mm}$ and the tensile speed $V_{z}=0.10 \mathrm{~mm} / \mathrm{min}$.

Table A1 The material parameters of aluminium pipe specimens and adhesive.

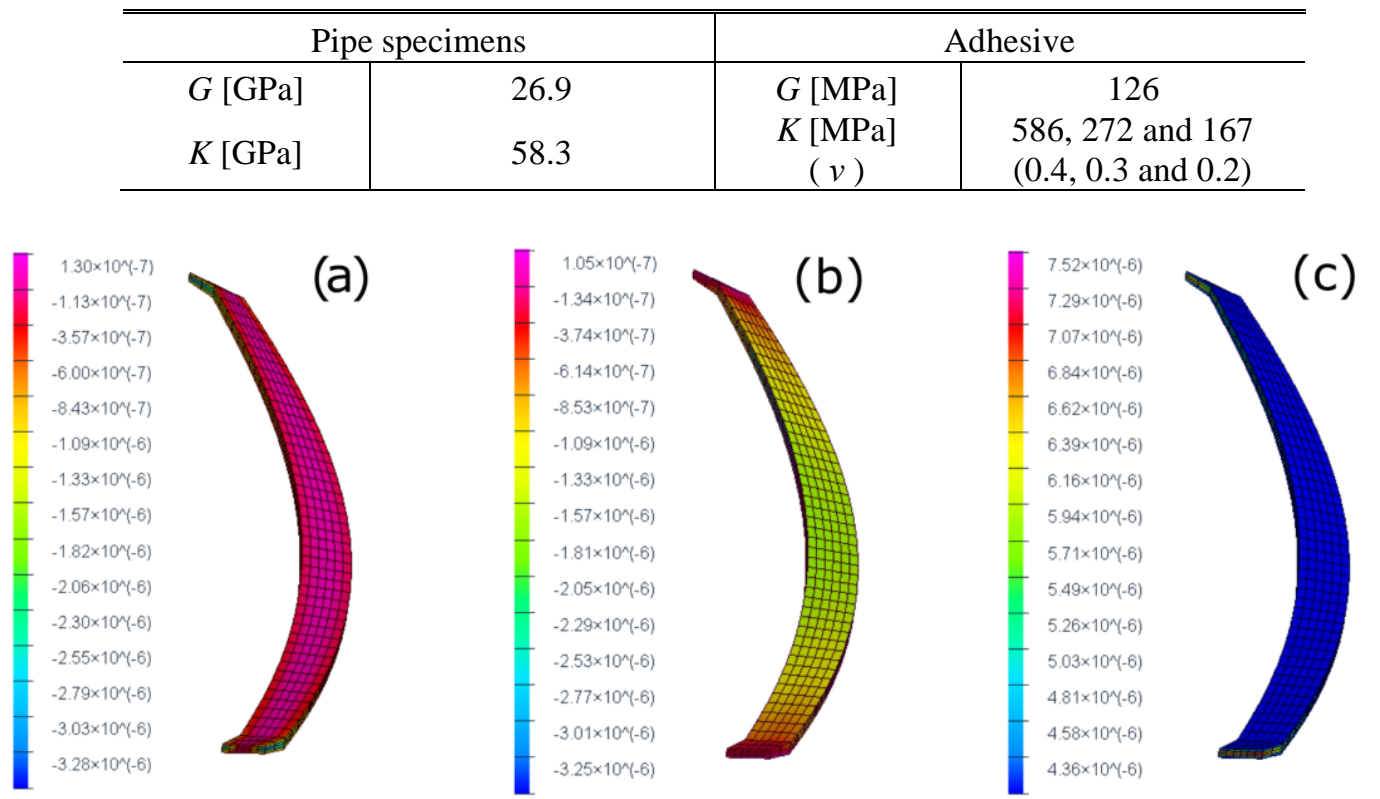

Fig. A2 The distribution of the strain tensor components (a) $\varepsilon_{r}$, (b) $\varepsilon_{\theta}$ and (c) $\varepsilon_{z}$ based on cylindrical coordinate in the adhesive layer. The parameters of adhesive are $G=126 \mathrm{MPa}$ and $K=586 \mathrm{MPa}(v=0.4)$.

Table A2 The ratio of averaged normal strains of $\varepsilon_{r^{\prime}}$ and $\varepsilon_{\theta^{\prime}}$ to the $\varepsilon_{z^{\prime}} \cdot v=0.4(G=126 \mathrm{MPa}, K=586 \mathrm{MPa})$ and $h=0.5 \mathrm{~mm}$.

\begin{tabular}{c|c|c|c|c}
\hline \hline & $0^{\circ}$ & $20^{\circ}$ & $45^{\circ}$ & $75^{\circ}$ \\
\hline$\varepsilon_{r^{\prime}} / \varepsilon_{z^{\prime}}$ & -0.17 & -0.15 & -0.14 & -0.16 \\
$\varepsilon_{\theta^{\prime}} / \varepsilon_{z^{\prime}}$ & -0.00 & -0.02 & -0.09 & -0.10 \\
\hline
\end{tabular}

Table A3 The ratio of averaged normal strains of $\varepsilon_{r^{\prime}}$ and $\varepsilon_{\theta^{\prime}}$ to the $\varepsilon_{z^{\prime}} \cdot v=0.3(G=126 \mathrm{MPa}, K=272 \mathrm{MPa})$ and $h=0.5 \mathrm{~mm}$.

\begin{tabular}{c|c|c|c|c}
\hline \hline & $0^{\circ}$ & $20^{\circ}$ & $45^{\circ}$ & $75^{\circ}$ \\
\hline$\varepsilon_{r^{\prime}} / \varepsilon_{z^{\prime}}$ & -0.09 & -0.08 & -0.07 & -0.09 \\
$\varepsilon_{\theta^{\prime}} / \varepsilon_{z^{\prime}}$ & -0.00 & -0.01 & -0.06 & -0.08 \\
\hline
\end{tabular}

Table A4 The ratio of averaged normal strains of $\varepsilon_{r^{\prime}}$ and $\varepsilon_{\theta^{\prime}}$ to the $\varepsilon_{z^{\prime}} . v=0.2(G=126 \mathrm{MPa}, K=167 \mathrm{MPa})$ and $h=0.5 \mathrm{~mm}$.

\begin{tabular}{c|c|c|c|c}
\hline \hline & $0^{\circ}$ & $20^{\circ}$ & $45^{\circ}$ & $75^{\circ}$ \\
\hline$\varepsilon_{r^{\prime}} / \varepsilon_{z^{\prime}}$ & -0.05 & -0.04 & -0.04 & -0.05 \\
$\varepsilon_{\theta^{\prime}} / \varepsilon_{z^{\prime}}$ & -0.00 & -0.01 & -0.05 & -0.07 \\
\hline
\end{tabular}




\section{Appendix B: Measuring displacement with image processing}

Figure B1 shows the adhesive layer of a plate specimen after a simple shear test. The red circle shows the location where we measured the displacement with a microscope and image processing analysis. We marked the plate specimen with a black line, and measured the distance between the edges of two separated segments of the black line in the tensile direction with shear displacement of the adhesive layer.

Figure B2 shows an example of a microscopic image of the adhesive layer during a tensile shear test. We used a median filter to remove noise and detected the edges of the mark using a Harris corner detector. In Fig. B2, we denote the distance from the upper edge of the mark in the left aluminium section to the lower edge in the right aluminium section as $d y$. We obtained the displacement of the adhesive layer as $d y-d y_{0}$, where $d y_{0}$ is the initial distance before the tensile shear test.

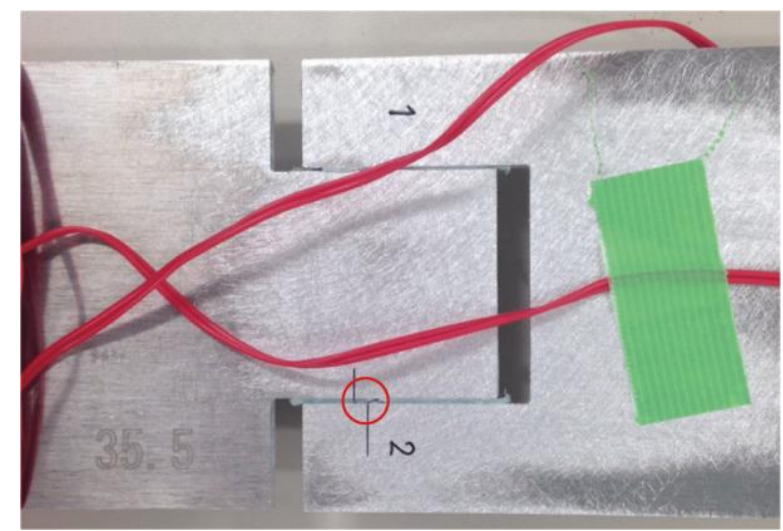

Fig. B1 Bonded view of a plate specimen. The red circle represents the location where we measured the adhesive displacement with a microscope.

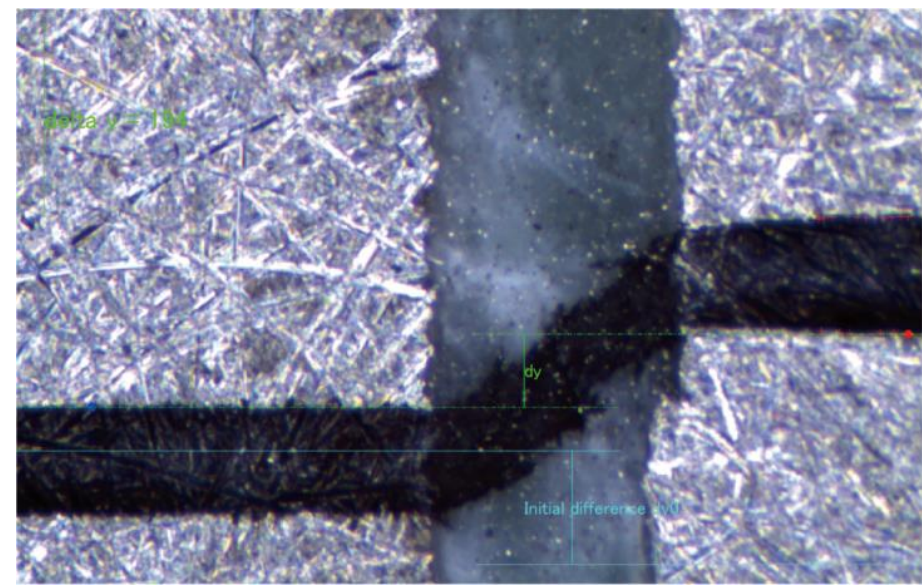

Fig. B2 Microscopic image of the adhesive layer during a tensile shear test $\left(h=0.62 \mathrm{~mm}, V_{z}=0.1 \mathrm{~mm} / \mathrm{min}\right)$. The shear displacement of the adhesive layer is calculated by subtracting the current difference $d y$ from the initial difference $d y_{0}$.

\section{References}

Adams, R. D., Strength predictions for lap joints, especially with composite adherends. A review, The Journal of Adhesion, Vol. 30, Nos. 1-4 (1989), pp. 219-242.

Adams, R. D., Atkins, R. W., Harris, and J. A., Kinloch, A. J., Stress analysis and failure properties of carbon-fibre-reinforced-plastic/steel double-lap joints, The Journal of Adhesion, Vol. 20, No. 1 (1986), pp. $29-53$.

Bossler, F. C., Franzblau, M. C., and Rutherford, J. L., Torsion apparatus for measuring shear properties of adhesive bonded joints, Journal of Physics E: Scientific Instruments, Vol. 1, No. 8 (1968), pp. 829-833. 
Brewer, J. C. and Lagace, P. A., Quadratic stress criterion for initiation of delamination, Journal of composite materials, Vol. 22, No.12 (1988), pp. 1141-1155.

Chai, H., Observation of deformation and damage at the tip of cracks in adhesive bonds loaded in shear and assessment of a criterion for fracture, International Journal of Fracture, Vol. 60, No. 4 (1993), pp. 311-326.

Chai, H., The effects of bond thickness, rate and temperature on the deformation and fracture of structural adhesives under shear loading, International journal of fracture, Vol. 130, No. 1 (2004), pp. 497-515.

Cognard, J. Y., Numerical analysis of edge effects in adhesively-bonded assemblies application to the determination of the adhesive behavior, Computers and Structures, Vol. 86, No. 17 (2008), pp. 1704-1717.

Cognard, J. Y., Davies, P., Gineste, B., and Sohier, L., Development of an improved adhesive test method for composite assembly design, Composites Science and Technology, Vol. 65, No. 3 (2005), pp. 359-368.

Cognard, J. Y., Davies, P., Sohier, L., and Créac'hcadec, R., A study of the non-linear behaviour of adhesively-bonded composite assemblies, Composite Structures, Vol. 76, No. 1 (2006), pp. 34-46.

da Silva, L. F. M., Rodrigues, T. N. S. S., Figueiredo, M. A. V., de Moura, M. F. S. F., and Chousal, J. A. G., Effect of adhesive type and thickness on the lap shear strength, The Journal of Adhesion, Vol. 82, No. 11 (2006) pp. 1091-1115.

Dolev, G. and Ishai, O., Mechanical characterization of adhesive layer in-situ and as bulk material, The Journal of Adhesion, Vol. 12, No. 4 (1981), pp. 283-294.

Green, R. J., A plasticity theory for porous solids, International Journal of Mechanical Sciences, Vol. 14, No. 4 (1972), pp. 215-224.

Ikegami, K. and Sugibayashi, T., A method of estimating the strength of adhesive bonded joints of metals, The Journal of Adhesion, Vol. 21, Nos. 3-4 (1987), pp. 211-227.

Kaelble, D. H., Theory and analysis of peel adhesion: adhesive thickness effects, The Journal of Adhesion, Vol. 37 , Nos. 1-3 (1992), pp. 205-214.

Katayama, S. and Kawahito, Y., Laser direct joining of metal and plastic, Scripta materialia, Vol. 59, No. 12 (2008), pp. 1247-1250.

Kawasaki, S., Nakajima, G., Haraga, K., and Sato, C., Functionally graded adhesive joints bonded by honeymoon adhesion using two types of second generation acrylic adhesives of two components, The Journal of Adhesion, Vol. 92, Nos. 7-9 (2016), pp. 517-534.

Liechti, K. M. and Hayashi, T., On the uniformity of stresses in some adhesive deformation specimens, The Journal of Adhesion, Vol. 29, Nos. 1-4 (1989), pp. 167-191.

Liyanage, T., Kilbourne, J., Gerlich, A. P., and North, T. H., Joint formation in dissimilar Al alloy/steel and Mg alloy/steel friction stir spot welds, Science and Technology of Welding and Joining, Vol. 14, No. 6 (2009), pp. 500-508.

Mahnken, R. and Schlimmer, M., Simulation of strength difference in elasto-plasticity for adhesive materials, International journal for numerical methods in engineering, Vol. 63, No. 10 (2005), pp. 1461-1477.

Pinto, A. M. G., Magalhães, A. G., Campilho, R. D. S. G., de Moura, M. F. S. F., and Baptista, A. P. M., Single-lap joints of similar and dissimilar adherends bonded with an acrylic adhesive, The Journal of Adhesion, Vol. 85, No. 6 (2009), pp. 351-376.

Richardson, D. E., McLennan, M. L., Anderson, G. L., Macon, D. J., and Batista-Rodriguez, A., Multiaxial, temperature, and time-dependent (matt) failure model, The Journal of Adhesion, Vol. 79, No. 2 (2003), pp. $157-174$.

Spaggiari, A., Castagnetti, D., and Dragoni, E., Mixed-mode strength of thin adhesive films: experimental characterization through a tubular specimen with reduced edge effect, The Journal of Adhesion, Vol. 89, No. 8 (2013), pp. 660-675.

Tanaka, H., Kimura, M., Iimori, M., Shibutani, Y., and Liu, Y., Failure Criteria of Adhesive Joints between Aluminum Circular Pipes under Multiaxial Stress State, Key Engineering Materials, Vol. 725 (2017), pp. 383-388.

Teutenberg, D., Meschut, G., Schlimmer, M., Kroll, U., and Matzenmiller, A., Testing, material data and identification of model parameters for toughened structural adhesives, Annual Meeting of the Adhesion Society (2013).

Thouless, M. D. and Jensen, H. M., Elastic fracture mechanics of the peel-test geometry, The Journal of Adhesion, Vol. 38, Nos. 3-4 (1992), pp. 185-197. 